\title{
Total Knee Arthroplasty Complicated by Partial Quadriceps Tendon Rupture: A Case Report
}

\author{
Ivan Chernev ${ }^{\mathrm{a}, \mathrm{b}, \mathrm{e}}$, George Orphanos ${ }^{\mathrm{c}}$, Lauren Chambers ${ }^{\mathrm{d}}$
}

\begin{abstract}
Disruption of the extensor mechanism after total knee arthroplasty is a rare but serious complication. It may involve the patellar tendon, patella or quadriceps tendon. Quadriceps tendon ruptures are less common and relatively few reports in the literature have addressed this complication. In particular, treatment guidelines for management of partial quadriceps rupture after total knee arthroplasty are lacking. Herein, we present the surgical technique and outcome of a patient with traumatic partial quadriceps tendon rupture after total knee arthroplasty using FiberTape and double figure of eight technique plus augmentation with TissueMend.
\end{abstract}

Keywords: Total knee arthroplasty; Partial quadriceps tendon rupture; Complication

\section{Introduction}

Disruption of the extensor mechanism after total knee arthroplasty (TKA) is a devastating postoperative complication, which may result in significant morbidity and functional limitations. It may involve the patellar tendon, patella or quadriceps tendon. Quadriceps tendon ruptures are less common and relatively few reports in the literature have ad-

Manuscript accepted for publication November 22, 2013

${ }^{\text {a}}$ Physical Medicine and Rehabilitation, West Virginia School of Osteopathic Medicine, Lewisburg, West Virginia, USA

${ }^{b}$ Physical Medicine and Rehabilitation, Beckley Appalachian Regional Healthcare, Beckley, West Virginia, USA

'Orthopedic Surgery, Beckley Appalachian Regional Healthcare, Beckley, West Virginia, USA

${ }^{\mathrm{d}}$ West Virginia School of Osteopathic Medicine, Lewisburg, West Virginia, USA

${ }^{\mathrm{e} C}$ Corresponding author: Ivan Chernev, ARH Southern WV Clinic, 250

Stanaford Road, Beckley, WV, 25801, USA.

Email: ivantchernev@yahoo.com

doi: http://dx.doi.org/10.4021/jmc1603w dressed this complication. The prevalence has been reported to range from $0.1 \%$ to $1.1 \%[1,2]$. Dobbs et al reported 24 $(0.1 \%)$ postoperative partial or complete quadriceps ruptures in a large cohort of 23,800 primary TKAs. Different surgical techniques have been described for repair of this challenging problem, however complications rates were high and outcomes were poor in many of these patients [1]. Singh et al described a technique using a double figure of eight Dacron tape for chronic rupture of the quadriceps tendon after TKA with an excellent outcome. He also advocated that with this technique early rehabilitation can be utilized [3]. Herein, we present the surgical technique and outcome of a patient with traumatic partial quadriceps tendon rupture after TKA, using FiberTape and similar double figure of eight technique plus augmentation with TissueMend.

\section{Case Report}

A 68-year-old woman with long-standing right knee pain due to osteoarthrosis underwent a primary TKA. Her previous medical history was significant for coronary artery disease and triple bypass surgery. She had an uneventful surgery and immediate postoperative period. She was discharged home on postoperative day six with home therapy. Ten days after the surgery she was seen in the office because she fell while walking outside of her home. She described that her knee was hyperflexed and abducted during the fall. When examined, she was not able to perform a straight leg raise test or active extension of the knee against gravity from sitting position, although minimal muscle movement was appreciated. X-ray of the knee showed the prosthesis and patella were both in good position; however damage to the extensor mechanism was suspected. Therefore, the patient was immobilized in full extension and an ultrasound of the knee was performed next day. The ultrasound revealed disruption of the extensor mechanism with a hematoma over the anterior medial side of the knee at the level of the musculotendinous junction, confirming a partial rupture of the quadriceps muscle tendon. Initially, the patient was treated conservatively and instructed to wear a knee immobilizer in full extension. However, the patient was not compliant with the immobilization and 


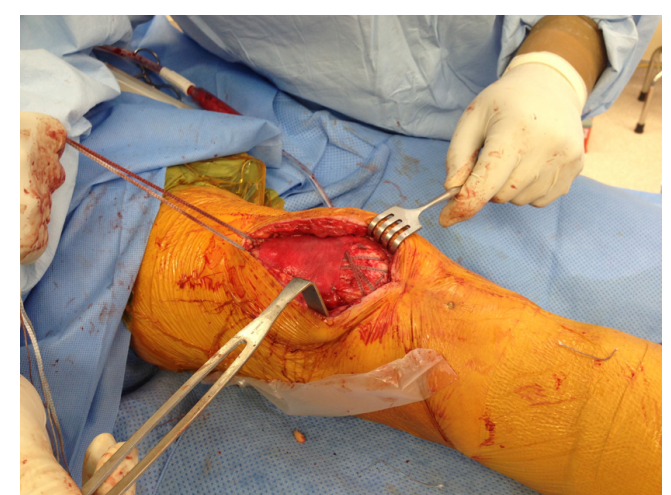

Figure 1. Intraoperative photograph of repaired partial quadriceps tendon rupture after total knee arthroplasty.

five weeks later she continued to have weakness, and was only able to actively extend the knee $25-30^{\circ}$ from a seated position. The decision was made to proceed with a surgical repair of the quadriceps tendon. The patient was prepped in the usual sterile fashion. Following the previous incision, the dissection was carried down to the extensor mechanism. The defective area filled with scar tissue was identified on the anteromedial aspect of the extensor tendon and retinaculum at the level of the musculotendinous junction. Therefore, the joint was entered through the scar tissue and irrigated. The edges were debrided and using \#2 FiberWire (Arthrex, Inc., Naples, FL, USA) an interrupted suture was performed. Additionally, $2 \mathrm{~mm}$ FiberTape (Arthrex, Inc., Naples, FL, USA) was doubled and passed through the proximal patellar tendon, then above the patella in figure of eight, and subsequently through the quadriceps tendon. Maintaining the knee in full extension the fiber tape was tightened. TissueMend (Sryker, Inc., Mahwah, NJ, USA) was sutured directly over the approximated area (Fig. 1).

The patient started isometric quadriceps muscle strengthening a few days after surgery. She was also given a knee immobilizer and instructed to use it for protected ambulation over the next few weeks. Six weeks after the surgery she had progressed to more aggressive physical therapy targeted at improving active knee range of motion (ROM) and strength. Seven months after the surgery the patient had quadriceps strength comparable to the contralateral side and full active ROM in extension.

\section{Discussion}

Rupture of the quadriceps tendon after TKA is an uncommon and serious complication; it may put significant technical challenges to the surgeon and be very demanding to the rehabilitation team and the patient. While primary quadriceps tendon repair in native knees has shown high success rates, repairs performed after TKA have not been as successful [1]. The literature is very limited regarding this uncommon but serious complication and consists only of case reports and case series. Different local and systemic factors such as previous knee surgery, lateral release, multiple steroid injections, rheumatoid arthritis, and diabetes mellitus have been suggested to play a role in the development of this complication.

As primary repair of the quadriceps tendon after TKA has not shown adequate results, different techniques using anchor sutures, auto and allografts, and synthetic materials augmentation have been described [3-7]. Furthermore, information regarding the treatment of partial ruptures is very limited with no clear guidelines of how to treat them. Although according to Dobbs et al non operative treatment of partial quadriceps tendon rupture has shown satisfactory results and fewer complications, it is not clear if more extensive ruptures have the same satisfactory rates.

We elected to perform the double figure of eight technique using FiberTape described above because it was technically easier and did not involve further disruption of the patella. Braided polyblend suture (FiberTape) is readily available in USA and is intended for use in soft tissue approximation. We used it to provide better approximation and strength instead of the Dacron tape described by Singh et al. In addition, we also opted to use soft tissue repair matrix (TissueMend). Soft tissue repair matrix is an acellular, collagen membrane used to reinforce soft tissues where weakness exists. It is specifically designed to serve as a scaffold for cellular and vascular ingrowth that is gradually remodeled into new tissue. It creates not only mechanical but also biological augmentation.

In conclusion, we believe that significant extent and near complete partial quadriceps tendon ruptures after TKA should be treated surgically similar to complete tears. One such technique is described above. As with complete ruptures, augmentation is necessary to prevent failure of the sutures. Soft tissue repair matrix may further augment the frail tissue area and possibly prevent re-ruptures.

\section{Conflicts of Interests}

The authors declare that there is no conflict of interests regarding the publication of this article.

\section{References}

1. Dobbs RE, Hanssen AD, Lewallen DG, Pagnano MW. Quadriceps tendon rupture after total knee arthroplasty. Prevalence, complications, and outcomes. J Bone Joint Surg Am. 2005;87(1):37-45.

2. Park SS, Kubiak EN, Wasserman B, Sathappan SS, Di Cesare PE. Management of extensor mechanism disruptions occurring after total knee arthroplasty. Am J Or- 
thop (Belle Mead NJ). 2005;34(8):365-372.

3. Singh S, Choon SK, Tai SS. A Modified Method for Reconstruction of Chronic Rupture of the Quadriceps Tendon after Total Knee Replacement. Malaysian Ortopaedic J. 2008;2(3):34-36.

4. Fernandez-Baillo N, Garay EG, Ordonez JM. Rupture of the quadriceps tendon after total knee arthroplasty. A case report. J Arthroplasty. 1993;8(3):331-333.

5. Pagnano MW. Patellar tendon and quadriceps ten- don tears after total knee arthroplasty. J Knee Surg. 2003;16(4):242-247.

6. Kim TW, Kamath AF, Israelite CL. Suture anchor repair of quadriceps tendon rupture after total knee arthroplasty. J Arthroplasty. 2011;26(5):817-820.

7. Lee YS, Min BH, Han KJ, Cho JH, Han SH, Lee DH, Oh KS. Simultaneous reconstruction of quadriceps tendon rupture after TKA and neglected Achilles tendon rupture. Orthopedics. 2010;33(5). 